\title{
STUDY PROTOCOL: INTENSIVE CARE ANXIETY AND EMOTIONAL RECOVERY (ICARE) - A PROSPECTIVE STUDY
}

Authors:

Maria I. Castillo E. RN, BN (Hons), Doctoral Candidate

Corresponding author: Griffith University, Nathan Campus, School of Nursing and Midwifery, Room 1.23 Building (N48), 170 Kessels Road, Nathan, Queensland 4111, Australia.

Tel.: +61 0731767291 ; fax: +61073176 7356 .

E-mail address: isabel.castillo@griffithuni.edu.au

Leanne M. Aitken RN, PhD, FRCNA, Professor of Critical Care Nursing Griffith University \& Princess Alexandra Hospital.

Postal address: Griffith University, Nathan Campus, School of Nursing and Midwifery (N48), 170 Kessels Road, Nathan, Queensland 4111, Australia.

Tel.: +61 073176 7256; fax: +61 073176 7356; E-mail address: l.aitken@griffith.edu.au

Marie L. Cooke RN, PhD, Professor School of Nursing and Midwifery, Griffith University Postal address: Griffith University, Nathan Campus, School of Nursing and Midwifery (N48), 170 Kessels Road, Nathan, Queensland 4111, Australia.

Tel.: +61 073735 5253; fax: +61 07373 57984; E-mail address: m.cooke@griffith.edu.au 
Keywords: Nursing, critical care, anxiety, depression, post-traumatic stress disorder.

\begin{abstract}
Background: Survivors of intensive care units (ICUs) commonly present with symptoms of anxiety, depression and post-traumatic stress disorder (PTSD) during recovery. A number of factors have been identified as predictors of these adverse emotional outcomes, but the role of state anxiety during critical illness in the development of these emotional problems remains unknown.

Purpose: The ICARe (Intensive $\underline{\text { Care }}$ Anxiety and Emotional Recovery) study protocol propose the development of an statistical model to determine the relationship between state anxiety during ICU stay and symptoms of anxiety, depression and PTSD at three occasions; after ICU discharge but prior to hospital discharge and at the third and sixth months post ICU discharge.
\end{abstract}

Methods: Prospective study including adult patients admitted to the ICU of a tertiary metropolitan Australian hospital for $\geq 24$ hours who are able to: (1) communicate verbally or nonverbally; (2) understand English and (3) open their eyes spontaneously or in response to voice to respond to the Faces Anxiety Scale (state anxiety assessment). One hundred and seventy patients will be assessed for their levels of state anxiety during their ICU stay to achieve a sample size of about 104 patients prior to hospital discharge. The outcomes of the ICARe study will include symptoms of anxiety, depression and PTSD assessed by standardised and well validated questionnaires widely used in intensive care research. Demographic, clinical, and social support information will also be collected.

Results: The projected sample size will provide sufficient power to evaluate the association between state anxiety and adverse emotional outcomes, as well as a variety of variables that will be entered into a multivariate regression analysis. 
Conclusion: This study will provide new evidence to improve care during critical illness and reduce adverse outcomes during recovery with the potential to decrease unnecessary suffering, promote comfort and improve long-term recovery. 


\section{Introduction}

Advances in research, technology and expert care have permitted continuous improvements in the survival of intensive care patients. However, these survivors commonly present with symptoms of anxiety, depression and post-traumatic stress disorder (PTSD) during recovery. Systematic reviews including studies of survivors of the ICU; mainly from developed countries such as the United Kingdom (UK), United States of America (USA), Australia and some European countries, have reported that the prevalence of these emotional problems in the ICU population is relatively high (1-3). When looking at the literature as a whole, it can be observed that approximately $25 \%$ of ICU survivors experience some emotional problem, either symptoms of anxiety, depression or PTSD. Further, it has been suggested that these emotional problems after the ICU experience may negatively affect these survivors' health-related quality of life (HRQoL) (4-9).

A number of factors have been identified as predictors of these adverse emotional outcomes in ICU survivors (7, 9-19). In the Intensive Care Anxiety and Emotional Recovery (ICARe) study, these risk factors have been classified into three categories: prior to critical illness, during intensive care treatment and after ICU discharge risk factors (Fig.1). Prior to critical illness risk factors include demographic characteristics such as female gender and younger age, low level of education, history of smoking and alcohol dependence, mental health history (premorbid anxiety, depression and psychiatric illness) and personality trait of pessimism. During intensive care risk factors include sedation, duration of mechanical ventilation, length of ICU stay and neurobiological factors such as hypoxaemia, hypoglycaemia and neuroendocrine dysregulation; and after ICU risk factors include memories of the ICU, neurocognitive impairment and lack of social support. The presence of state anxiety during ICU stay has been identified as a frequent and serious problem for critically ill patients, which could be another possible during intensive care treatment factor, related to adverse emotional outcomes, however, the relationship 
between this emotion during critical illness and adverse emotional outcomes remains unknown (20).

Anxiety was conceptualised as state anxiety and trait anxiety by Spielberger in the sixties (21). This distinction has provided a better understanding of this complex phenomenon and this approach has been recognised in much literature and research. State anxiety is defined as a normal and temporary emotion that involves physiological arousal and feelings of tension, apprehension, nervousness and worry when a stressful situation is perceived. Trait anxiety, on the other hand, corresponds to the person's tendency to become state anxious as part of their personality trait (22). Because critically ill patients are constantly exposed to a great variety of stressors while receiving intensive care treatment, state anxiety is highly prevalent in the ICU population, especially in those requiring mechanical ventilation (23). In Australia, for example, an investigation including $106 \mathrm{ICU}$ patients found some level of anxiety in $85 \%$ of them (24). The level of anxiety reported was moderate to severe despite receiving sedation and/or analgesia (24).

In addition, it is known that anxiety during critical illness is either poorly assessed or not assessed at all because of the challenge implied in assessing a self-report symptom in patients with inability to verbalise their feelings due to endotracheal intubation and mechanical ventilation. Thus, clinicians often identify state anxiety by observation of behavioral (e.g. restlessness) and physiological (e.g. tachycardia) manifestations (24, 25). Unfortunately, clinicians' observations of these two components are unreliable indicators of state anxiety in ICU settings since common conditions such as delirium or pain share similar physiological and behavioural characteristics with this emotion which may lead to erroneous symptom interpretation (25). Moreover, it has been found that state anxiety may not always be accompanied by physiological changes $(24,26,27)$. 
Another factor that has made the assessment difficult is the lack of appropriate instruments to assess state anxiety in ICU patients. Only recently, specially designed tools for the assessment of this emotion in seriously ill patients have been available, although the use of these has not yet been included in routine clinical practice $(24,27-29)$. This study protocol proposes the development of a statistical model in order to acquire a better understanding about the relationship between state anxiety during the ICU stay and short and medium-term emotional outcomes in survivors of the ICU as well as the association between the levels of state anxiety and sedation/analgesia during the intensive care treatment.

\section{Methods}

\section{Aims}

This study aims to determine the association between state anxiety during the ICU stay and symptom of anxiety, depression and PTSD at three occasions in survivors of the ICU; after ICU discharge but prior to hospital discharge and at the third and sixth months after ICU discharge. It also aims to examine the relationship between state anxiety and sedation/analgesia for ICU patients during the intensive care treatment.

\section{Design}

This research is a prospective longitudinal cohort study of ICU survivors. This observational design will allow studying state anxiety during the ICU stay as an intra-ICU risk factor for the subsequent development of adverse emotional outcomes during recovery. In addition, it will also allow follow up of the patients at three occasions to determine the short and medium-term effects of state anxiety on emotional recovery.

\section{Setting}


This study will be conducted in the adult ICU of a tertiary metropolitan hospital located in Brisbane, Queensland, Australia. This ICU has 25 beds, including general ICU patients and post-cardiac surgery patients. The nurse/patient ratio is $1: 1$ and in $2009 / 2010$, there were approximately 2000 admissions to this ICU.

\section{$\underline{\text { Sample }}$}

All adult patients ( $\geq 18$ years of age) admitted to the ICU for $\geq 24$ hours, who are able to: (1) communicate verbally or non-verbally; (2) understand English; and, (3) open their eyes spontaneously or in response to voice to respond to the Faces Anxiety Scale (FAS) will be invited to participate in this study.

\section{Sample size}

In this study, previous research exploring similar research question in the ICU population has been examined to determine the effect size reported and perform power analysis a priori using G*Power $3.1 .3(30)$. The effect size reported in the literature testing correlations in the ICU context is often medium (1-3). Therefore, a medium effect size and a selected power of $80 \%$ with a significance of $\alpha=0.05$ have been used to estimate the sample size for this study.

In addition, the variables that are significant at a 0.1 level will be entered into a multivariate regression analysis. The number of predictors that will be entered into this model is unknown at this stage, but based on the number of prior to critical illness, during intensive care treatment and after ICU discharge variables that will be controlled for; the number of predictors is expected to be seven. By convention, a moderate effect size for multiple regression is $R^{2}=0.13$, therefore, using power of $80 \%$ and $\alpha=0.05$, the number of participants needed is $104(31,32)$.

Because longitudinal research in the ICU population often reports a number of patients lost to follow up, 170 patients will be assessed for state anxiety during their ICU stay to obtain a sample 
of about 104 patients prior to hospital discharge. We have estimated $70 \%$ completion of follow up prior to hospital discharge and an in-hospital mortality rate of $10 \%$.

\section{$\underline{\text { Recruitment process }}$}

All ICU patients will be screened daily by the principal investigator for potential enrolment, with liaison with the ICU Research Nurse to determine eligibility. Each participant will be approached at an appropriate time determined in consultation with the bed-side ICU nurse. Patients' assent will be required to include patients in this study during the ICU stay, and then when the patients are in the wards (after the ICU stay but prior to hospital discharge), written consent will be requested. This process has been approved by the relevant Human Research Ethics Committees.

\section{Data collection}

Data regarding prior to critical illness, during intensive care treatment and after ICU discharge variables will be collected in order to control for confounding variables when testing the association between state anxiety and adverse emotional outcomes. All the data needed for this study will be collected within nine months from the commencement of this study, at four occasions; (1) during the ICU stay, (2) after the ICU stay but prior to hospital discharge, (3) at the third and (4) sixth months after hospital discharge.

During the patients' ICU stay, the levels of state anxiety will be assessed according to a protocol, and clinical and demographic data will be collected from medical records. Data regarding adverse emotional outcomes and some confounding variables such as memories of the ICU, cognitive functioning, personality trait of optimism versus pessimism, personality trait of anxiety, smoking and alcohol consumption and social support will be assessed using standardised questionnaires. These questionnaires will be administered after the ICU stay but prior to hospital discharge and at the third and sixth month after ICU discharge. The questionnaires will contain 
approximately 67 items, and will be given to the patient with a covering letter providing instructions for participants to complete the questionnaires within the next days at a time convenient to them, but without assistance from family members/friends. At the third and sixth months post ICU discharge, the same questionnaires, with the exception of personality trait of optimism versus pessimism and personality trait of anxiety, will be posted to the participants' address. An appointment for a phone interview will be scheduled so participants can read their answers to the researcher. Alternatively, a reply paid envelope will be provided. The researcher will contact the participant or next of kin to confirm that the participants are alive before posting all the questionnaires.

Mortality during the ICU stay, at the third month and sixth month after ICU discharge will be recorded. Follow-up at the three occasions proposed in this study will enable the assessment of the impact of state anxiety during the ICU stay on medium and long-term emotional recovery and fluctuations of this relationship over time.

\section{$\underline{\text { Variables }}$}

Prior to critical illness, during intensive care treatment and after ICU discharge variables, the relevant measure and the time point for assessment are summarised in tables 1 and 2 . The assessment of state anxiety will be explained in more detail since this is the primary predictive variable of interest in this study.

\section{$\underline{\text { State anxiety assessment tool }}$}

Self- reported levels of state anxiety during the ICU stay will be assessed by the Faces Anxiety Scale (FAS) (24). The FAS is a single-item instrument especially designed to measure state anxiety in ICU settings. It consists of a scale showing five faces representing five different levels of anxiety, ranging from no anxiety to extreme anxiety, scoring from one to five. The patient is 
asked how much anxiety is felt at the moment of assessment and the answer may by a verbal or a nonverbal response, i.e. they can point to the relevant face. The reported criterion validity of this tool was $0.64(P<0.001)$ in mechanically ventilated patient (correlation between the selfreport of anxiety on the FAS and clinical judgment of anxiety) (24). While its reliability has not been measured because of limitations in reliability methods for a single-item instrument $(24,33)$, the FAS is a practical tool to assess this emotion in critically ill patients.

\section{State anxiety assessment protocol}

After screening for eligible participants with the ICU research nurse, the researcher and the bedside nurse will discuss and plan the best time for the assessment. State anxiety will be assessed twice daily (morning $8-11 \mathrm{am}$ and evening $4-7 \mathrm{pm}$ ). The time to perform this assessment is approximately one minute and does not involve any invasive procedure. After the bed-side nurse agrees, the researcher will observe and record the airway status (tracheostomy, endotracheal tube), mechanical ventilation status (invasive, non-invasive, non-ventilation), delirium status (The Confusion Assessment Method for the ICU), oxygen saturation, pain score (Verbal Pain Scale and/or The Critical Care Pain Observation Tool) and main sedation administration method (continuous infusion, intermittent, daily sedative interruption). Then, the researcher will approach the patient and explain the procedure. The patient will be shown the Faces Anxiety Scale (FAS) with the following instructions:

- These faces are showing different levels of anxiety.

- This face shows no anxiety at all; this face shows a little bit more; a bit more (sweep finger along scale); right up to extreme anxiety.

- Have a look at these faces and choose the one that shows how much anxiety you feel at the moment (34). 
The level of state anxiety will be measured as a continuous variable (scale from 1 to 5 points), and then the value obtained will be categorised into, low (1 -2) or moderate to severe anxiety (score 3-5) for analysis, consistent with instructions provided by the scale developer.

\section{$\underline{\text { Adverse Emotional Outcomes }}$}

The adverse emotional outcomes assessed in this study include symptoms of anxiety, depression and PTSD measured at three occasions, after ICU discharge but before hospital discharge, at the third and sixth month after ICU discharge. These outcomes will be assessed by standardised self-report measures, widely used in ICU research.

Symptoms of anxiety and depression will be measured by the Hospital Anxiety Depression Scale (HADS) (35). This self-report questionnaire consists in 14 statements divided into two sevenstatement subscales, one for depression and one for anxiety. Each statement has four possible alternatives (0-3) indicating the frequency of the feeling assessed. Only one alternative is chosen for each statement. Three scoring ranges for each subscale are possible: normal (0-7), borderline abnormal (8-10, suggesting possible psychological distress) and abnormal (11-21, suggesting probable psychological distress). Symptoms of anxiety and depression will be classified into the three categories of the HADS recommended in Zigmond\&Snaith (1983). These categories are normal (score 0-7), possible case (score 8-10) and probably case (score 11-21). The HADS assesses symptoms of anxiety and depression in hospital settings, and it is commonly used as an instrument to measure these adverse emotional outcomes in critical care research (36-39).

Symptoms of post-traumatic stress disorder will be measured by the Post-Traumatic Stress Syndrome 10-Questions Inventory (PTSS-10) (40). This is a self-report two-part questionnaire (part A and part B) designed to screen for PTSD symptoms in survivors of critical illness. Part A 
consists in a statement about the patients' memory of their ICU admission. Two alternatives (Yes/ No) are possible to indicate the presence or absence of four traumatic memories. These memories are memories of nightmares; severe anxiety or panic; severe pain; and, respiratory distress. In part B, the presence and intensity of 10 PTSD symptoms are assessed. These symptoms are sleeping disturbance, nightmares, depression, hyperalertness, emotional numbing, irritability, labile mood, guilt, avoidance of activities prompting recall of the traumatising event, and muscular tension. Each symptom can be rated from 1 (never) to 7 (always); therefore, a total score ranging from 10 to 70 points can be obtained. Symptoms of PTSD will be classified according the recommended cut-off of 35 points (scale from 10 to 70 points) into two categories, high probability of PTSD (total score $>35$ points) and low probability of PTSD $(<35$ points) (40).

\section{Planned analyses}

The Statistical Package for the Social Sciences version 20.0 (SPSS IBM Corp. in Armonk, NY) will be used to analyse the data. Once the data have been entered into SPSS, all variables will be cleaned and checked for missing and invalid values. Following data cleaning a $15 \%$ random sample of the database will be verified against original questionnaires (this will be conducted for all data collection points). Descriptions of categorical variables will be presented as numbers and percentages. Continuous data will be presented as a mean $( \pm S D)$ or median with interquartile range. Appropriate statistical tests will be used to test bivariate relationships between the dependent variables and the independent variables. Continuous/Interval variables will be tested to confirm normality distribution before deciding on either parametric analysis (independent ttest, one-way ANOVA, Pearson's r) and/or non-parametric analysis (Mann-Whitney U, Kruskal Wallis \& Spearman's). Chi-square tests will be performed for categorical data. When considering the analysis of longitudinal data, appropriate repeated measures analyses will be employed. Variables associated with $p<0.10$ on bivariate analysis will be included in the final 
models to identify which factors are independently associated with adverse emotional outcomes (symptoms of anxiety, depression and PTSD). A stepwise process will be used during the model building, where variables not reaching a significance level $p<0.05$ will be omitted. Modelling of longitudinal data will involve mixed effects analysis of emotional outcomes over time.

\section{Ethical Considerations}

This study has been approved by the Hospital and Griffith University Human Research Ethics Committees. Verbal or nonverbal assent will be sought from each patient during the ICU stay prior to collection of data. Then, when the patients are in the wards, written informed consent will be sought from each patient. Patients, who do not give consent, will be asked permission to use the ICU based-data. If permission to use this information is not given, the data will be destroyed. All data will be stored in locked facilities, with identifying and contact details stored separately to study data. Publications and presentations will be prepared in such a manner as to maintain the confidentiality and anonymity of all study participants. In this study, the general values and principles set out in the National Statement on Ethical Conduct in Human Research will be applied (41).

\section{Results and discussion}

The ICARe study is a single-center prospective longitudinal study that intends to address the issue of adverse emotional outcomes in survivors of the ICU. Whereas previous studies have measured short, medium and long-term emotional outcomes and identified factors that may lead to the development of anxiety, depression and PTSD in ICU survivors, the impact of the amount of state anxiety that patients feel during the ICU stay on emotional recovery remains unclear.

To date, there is enough evidence to propose an existing relationship between state anxiety during the ICU stay and adverse emotional outcomes during recovery. Patients who present with moderate to severe levels of state anxiety during critical illness may be at a higher risk for 
developing symptoms of anxiety, depression and PTSD during recovery than those with low or no anxiety. This relationship may be through a direct link and/or an indirect link involving sedation. As the emotional component of state anxiety is poorly assessed during critical illness, state anxiety might not be detected in a timely manner and, therefore, remain unrelieved for long periods of time. This situation may have a direct impact on the development of adverse emotional outcomes in the longer term. On the other hand, if anxiety progresses to extreme levels and patients exhibit psychomotor agitation, the use of increased doses of sedative agents may be given. Thus, sedation could represent an indirect link to adverse emotional outcomes after the ICU experience.

The significance of this study can be considered from a number of viewpoints. The first aspect concerns ICU survivors and their families. For many patients, the presence of these emotional consequences can impact their quality of life, affecting for example, their family and social relationships; employment and economic status; and physical recovery.

The second aspect relates to health care professionals. State anxiety is widely acknowledged as a serious problem in ICU settings despite current ICU management. However, the lack of evidence-based knowledge on the consequences that this emotion may have on the short, medium and long-term emotional recovery of patients, may lead clinicians to disregard the assessment and management of this emotion during ICU treatment. The ICARe study seeks to understand this association and inform clinicians about the short and medium-term effects of state anxiety on emotional recovery, so as to facilitate change in clinical practice and improve outcomes for ICU survivors.

The third aspect pertains to the efficient use of health care resources. Systematic reviews, with an international viewpoint, have estimated that approximately a quarter of the patients who survive the intensive care treatment develop emotional problems, which may endure for years (1-3). The emotional recovery of these patients involves health care resources, expenses that 
could be avoided by preventing these emotional consequences. Considering that annually millions of patients are admitted to ICUs around the world, this situation is, undoubtedly, an important public health issue. Ultimately, the findings of this study will generate new evidence to inform future research and clinical practice.

The strength of the ICARe study is that a comprehensive assessment of the most relevant prior to critical illness, during intensive care treatment and after ICU discharge factors described in the literature will be conducted. This strength will permit the development of a conceptual model where the significance of the relationships between risk factors and adverse emotional outcomes will be identified. In addition, the direction of these associations will be determined.

The ICARe study proposal has some constraints. State anxiety will be assessed after 24 hours of ICU admission, twice daily during the patients' ICU stay. Therefore, it is possible that, despite frequent data collection, the ICARe study may not fully capture the variations of the levels of anxiety within a 24 hour period. The levels of state anxiety may vary over time in a dynamic environment such as the ICU. Nevertheless, this emotion could only be assessed twice daily due to the burden associated with the data collection process. Although the design of the ICARe study includes a comprehensive collection of prior to critical illness, during intensive care treatment and after ICU discharge variables that will take into account confounding variables and bias, so that the effects of state anxiety on adverse emotional outcomes can be determined, some key risk factors for the development of these adverse emotional outcomes may still be unknown or unmeasured in this study.

\section{Conclusion}

The ICARe study is a prospective longitudinal study of survivors of ICU treatment six months after discharge. This study aims to provide new knowledge about the association between state anxiety during the ICU stay and the development of symptoms of anxiety, depression and PTSD 
during recovery. The strengths of the study include comprehensive measurement of prior to critical illness, during intensive care treatment and after ICU discharge risk factors for the development of relevant adverse emotional outcomes; an adequate sample size, determined by power analysis; and the development of a statistical model. This research has the potential to inform the development of interventions designed to improve the emotional outcomes of patients being admitted to intensive care units by describing factors that affect their recovery. Improved emotional recovery may result in reduced burden for patients, their families and the health care system. 


\section{References}

1. Davydow DS, Desai SV, Needham DM, Bienvenu OJ. Psychiatric morbidity in survivors of the acute respiratory distress syndrome: a systematic review. Psychosomatic Medicine 2008;70(4):512-9.

2. Davydow DS, Gifford JM, Desai SV, Needham DM, Bienvenu OJ. Posttraumatic stress disorder in general intensive care unit survivors: a systematic review. General Hospital Psychiatry 2008;30(5):421-34.

3. Davydow DS, Gifford JM, Desai SV, Bienvenu OJ, Needham DM. Depression in general intensive care unit survivors: a systematic review. Intensive Care Medicine 2009;35(5):796-809.

4. Weinert CR, Gross CR, Kangas JR, Bury CL, Marinelli WA. Health-related quality of life after acute lung injury. American Journal of Respiratory and Critical Care Medicine 1997;156(4 Pt 1):1120-8.

5. Hopkins RO, Orme JF, Jr., Weaver LK, Chan KJ. Quality of life, emotional, and cognitive function following acute respiratory distress syndrome. Journal of the International Neuropsychological Society 2004;10(7):1005.

6. Kapfhammer HP, Rothenhausler HB, Krauseneck T, Stoll C, Schelling G. Posttraumatic stress disorder and health-related quality of life in long-term survivors of acute respiratory distress syndrome. The American Journal of Psychiatry 2004;161(1):45-52.

7. Deja M, Denke C, Weber-Carstens S, Schröder J, Pille C, Hokema F, Falke K, Kaisers U. Social support during intensive care unit stay might improve mental impairment and consequently health-related quality of life in survivors of severe acute respiratory distress syndrome. Critical Care 2006;10(5):147.

8. Sukantarat K, Greer S, Brett S, Williamson R. Physical and psychological sequelae of critical illness. British Journal Of Health Psychology 2007;12(Pt 1):65-74.

9. Girard TD, Shintani AK, Jackson JC, Gordon SM, Pun BT, Henderson MS, Dittus RS, Bernard GR, Ely EW. Risk factors for post-traumatic stress disorder symptoms following critical illness requiring mechanical ventilation: a prospective cohort study. Critical Care 2007;11(1):28.

10. Jones C, Bäckman C, Capuzzo M, Flaatten H, Rylander C, Griffiths RD. Precipitants of post-traumatic stress disorder following intensive care: a hypothesis generating study of diversity in care. Intensive Care Medicine 2007;33(6):978-85.

11. Cuthbertson BH, Hull A, Strachan M, Scott J. Post-traumatic stress disorder after critical illness requiring general intensive care. Intensive Care Medicine 2004;30(3):450-5.

12. Hopkins RO, Key CW, Suchyta MR, Weaver LK, Orme JF, Jr. Risk factors for depression and anxiety in survivors of acute respiratory distress syndrome. General Hospital Psychiatry 2010;32(2):147-55.

13. Rattray JE, Johnston M, Wildsmith JA. Predictors of emotional outcomes of intensive care. Anaesthesia 2005;60(11):1085-92.

14. Granja C, Gomes E, Amaro A, Ribeiro O, Jones C, Carneiro A, Costa-Pereira A. Understanding posttraumatic stress disorder-related symptoms after critical care: The early illness amnesia hypothesis. Critical Care Medicine 2008;36(10):2801-9.

15. Schelling G, Briegel J, Roozendaal B, Stoll C, Rothenhausler HB, Kapfhammer HP. The effect of stress doses of hydrocortisone during septic shock on posttraumatic stress disorder in survivors. Biological Psychiatry 2001;50(12):978-85.

16. Myhren H, Ekeberg $\varnothing$, Tøien K, Karlsson S, Stokland O. Posttraumatic stress, anxiety and depression symptoms in patients during the first year post intensive care unit discharge. Critical Care 2010;14(1):R14.

17. Nelson BJ, Weinert CR, Bury CL, Marinelli WA, Gross CR. Intensive care unit drug use and subsequent quality of life in acute lung injury patients. Critical Care Medicine 2000;28(11):3626-30.

18. Jackson JC, Hart RP, Gordon SM, Shintani A, Truman B, May L, Ely EW. Six-month neuropsychological outcome of medical intensive care unit patients. Critical Care Medicine 2003;31(4):1226-34.

19. Dowdy DW, Dinglas V, Mendez-Tellez PA, Bienvenu J, Sevransky J, Dennison CR, Shanholtz C, Needham DM. Intensive care unit hypoglycemia predicts depression during early recovery from acute lung injury. Critical Care Medicine 2008;36(10):2726-33. 
20. Kress JP, Gehlbach B, Lacy M, Pliskin N, Pohlman AS, Hall JB. The long-term psychological effects of daily sedative interruption on critically ill patients. American Journal of Respiratory \& Critical Care Medicine 2003;168(12):1457-61.

21. Spielberger CD. Theory and research on anxiety. In: Spielberger CD, editor. Anxiety and behavior. New York: Academic Press; 1966. p. 3-20.

22. Spielberger CD, Reheiser EC. Assessment of Emotions: Anxiety, Anger, Depression, and Curiosity. Applied Psychology : Health and Well-Being 2009;1(3):271-302.

23. Chlan LL. Description of anxiety levels by individual differences and clinical factors in patients receiving mechanical ventilatory support. Heart \& Lung 2003;32(4):275-82.

24. McKinley S, Stein-Parbury J, Chehelnabi A, Lovas J. Assessment of anxiety in intensive care patients by using the Faces Anxiety Scale. American Journal of Critical Care 2004;13(2):146-52.

25. Tate JA, Devito Dabbs A, Hoffman LA, Milbrandt E, Happ MB. Anxiety and Agitation in Mechanically Ventilated Patients. Qualitative Health Research 2012;22(2):157-73.

26. De Jong MJ, Moser DK, An K, Chung ML. Anxiety is not manifested by elevated heart rate and blood pressure in acutely ill cardiac patients. European Journal of Cardiovascular Nursing 2004;3(3):247-53.

27. De Jong MJ, Burns SM, Campbell ML, Chulay M. Development of the American Association of Critical-Care Nurses' Sedation Assessment Scale for Critically III Patients. American Journal of Critical Care 2005;14(6):531--44.

28. Moser DK, Chung ML, McKinley S, Riegel B, An K, Cherrington CC, Blakely W, Biddle M, Frazier SK, Garvin BJ. Critical care nursing practice regarding patient anxiety assessment and management. Intensive \& Critical Care Nursing 2003;19(5):276-88.

29. O'Brien JL, Moser DK, Riegel B, Frazier SK. Comparison of anxiety assessments between clinicians and patients with acute myocardial infarction in cardiac critical care units. American Journal of Critical Care 2001;10(2):97--103.

30. Faul F, Erdfelder E, Buchner A, Lang A-G. Statistical power analyses using G Power 3.1: Tests for correlation and regression analyses. Behavior Research Methods 2009;41(4):1149-60.

31. Polit-O'Hara D, Beck CT. Nursing research: generating and assessing evidence for nursing practice. Philadelphia: Wolters Kluwer/Lippincott Williams \& Wilkins; 2012.

32. Polit-O'Hara D. Statistics and data analysis for nursing research. Boston: Pearson; 2010.

33. McKinley S, Madronio C. Validity of the Faces Anxiety Scale for the assessment of state anxiety in intensive care patients not receiving mechanical ventilation. Journal of Psychosomatic Research 2008;64(5):503-7.

34. McKinley S, Coote K, Stein-Parbury J. Development and testing of a Faces Scale for the assessment of anxiety in critically ill patients. Journal of Advanced Nursing 2003;41(1):73-9.

35. Zigmond AS, Snaith RP. The Hospital Anxiety and Depression Scale. Acta Psychiatrica Scandinavica 1983;67(6):361-70.

36. Jones C, Griffiths RD, Humphris G, Skirrow PM. Memory, delusions, and the development of acute posttraumatic stress disorder-related symptoms after intensive care. Critical Care Medicine 2001;29(3):573-80.

37. Jones $C$, Skirrow P, Griffiths RD, Humphris GH, Ingleby S, Eddleston J, Waldmann C, Gager M. Rehabilitation after critical illness: A randomized, controlled trial. Critical Care Medicine 2003;31(10):245661.

38. Rattray J, Johnston M, Wildsmith JAW. The intensive care experience: development of the ICE questionnaire. Journal of Advanced Nursing 2004;47(1):64-73.

39. Scragg P, Jones A, Fauvel N. Psychological problems following ICU treatment. Anaesthesia 2001;56(1):9-14.

40. Stoll C, Kapfhammer HP, Rothenhäusler HB, Haller M, Briegel J, Schmidt M, Krauseneck T, Durst K, Schelling G. Sensitivity and specificity of a screening test to document traumatic experiences and to diagnose post-traumatic stress disorder in ARDS patients after intensive care treatment. Intensive Care Medicine 1999;25(7):697-704.

41. NHMRC A, AVCC. National statement on ethical conduct in human research. 2007 [14th January 2012]; Available from: http://www.nhmrc.gov.au/publications/synopses/ files/e72.pdf 
Table 1: study constructs, measures, number of items and data collection schedule

\begin{tabular}{|c|c|c|c|c|c|}
\hline Measure & Outcomes & ICU & $\begin{array}{l}\text { After ICU, but prior to } \\
\text { hospital discharge }\end{array}$ & $3^{\text {rd. }} \mathrm{mo}$. & $6^{\text {th. }} \mathrm{mo}$. \\
\hline HADS & Symptoms of anxiety & & 7 & 7 & 7 \\
\hline HADS & Symptoms of depression & & 7 & 7 & 7 \\
\hline Part B of PTSS-10 & Symptoms of PTSD & & & 10 & 10 \\
\hline Measure & Variables & & & & \\
\hline FAS & State anxiety & 1 & & & \\
\hline Part A PTSS-10 & Memory & & 1 & 1 & 1 \\
\hline$\overline{L O T-R}$ & Trait pessimism & & 6 & & \\
\hline STAI Form Y-2 & Trait anxiety & & 20 & & \\
\hline MSPSS & Social support & & 12 & 12 & 12 \\
\hline MOS COG-R & Cognitive function & & 6 & 6 & 6 \\
\hline QF method & Smoking & & 2 & 2 & 2 \\
\hline GQF method & Alcohol consumption & & 2 & 2 & 2 \\
\hline \multirow{4}{*}{$\begin{array}{l}\text { Patient } \\
\text { demographics } \\
\text { questionnaire }\end{array}$} & Level of education & & 1 & & \\
\hline & Employment status & & 1 & 1 & 1 \\
\hline & Mental health history & & 1 & & \\
\hline & $\begin{array}{l}\text { Pre-ICU medications } \\
\text { (steroids, opioids, benzos, } \\
\text { beta-blockers, anxiolytics) }\end{array}$ & & 1 & & \\
\hline Total No of Items & & 1 & 67 & 48 & 48 \\
\hline
\end{tabular}

HADS: Hospital Anxiety Depression Scale; PTSS-10: Post-Traumatic Stress Syndrome 10-Questions Inventory; PTSD: Post-traumatic Stress Disorder; FAS: Faces Anxiety Scale; LOT-R: Revised Life Orientation Test; STAI Form Y-2: State Trait-Anxiety Inventory Form Y-2; MSPSS: The Multidimensional Scale of Social Support; MOS COG-R: Medical Outcomes Study Cognitive Functioning Scale-Revised; QF method: Quantity Frequency method; GQF method: Graduated Quantity Frequency method; APACHE III: Acute Physiology and Chronic Health Evaluation III. 
Table 2: clinical and demographic data, collected from medical records during the ICU stay

\begin{tabular}{|l|}
\hline Gender \& age \\
\hline Airway status (tracheostomy, endotracheal intubation) \\
\hline Hypoxaemia (oxygen saturation) \\
\hline Diagnosis ICU admission \\
\hline Severity of illness: Acute Physiology and Chronic Health Evaluation III (APACHE III) \\
\hline Delirium \\
\hline Length of mechanical ventilation, ICU \& hospital stay \\
\hline MV status (invasive, non-invasive, non-ventilation) \\
\hline Sedation administration method (continuous, intermittent, daily sedative interruption) \\
\hline Duration of sedation \& total doses of sedatives, opioids, steroids and anxiolytics \\
\hline
\end{tabular}




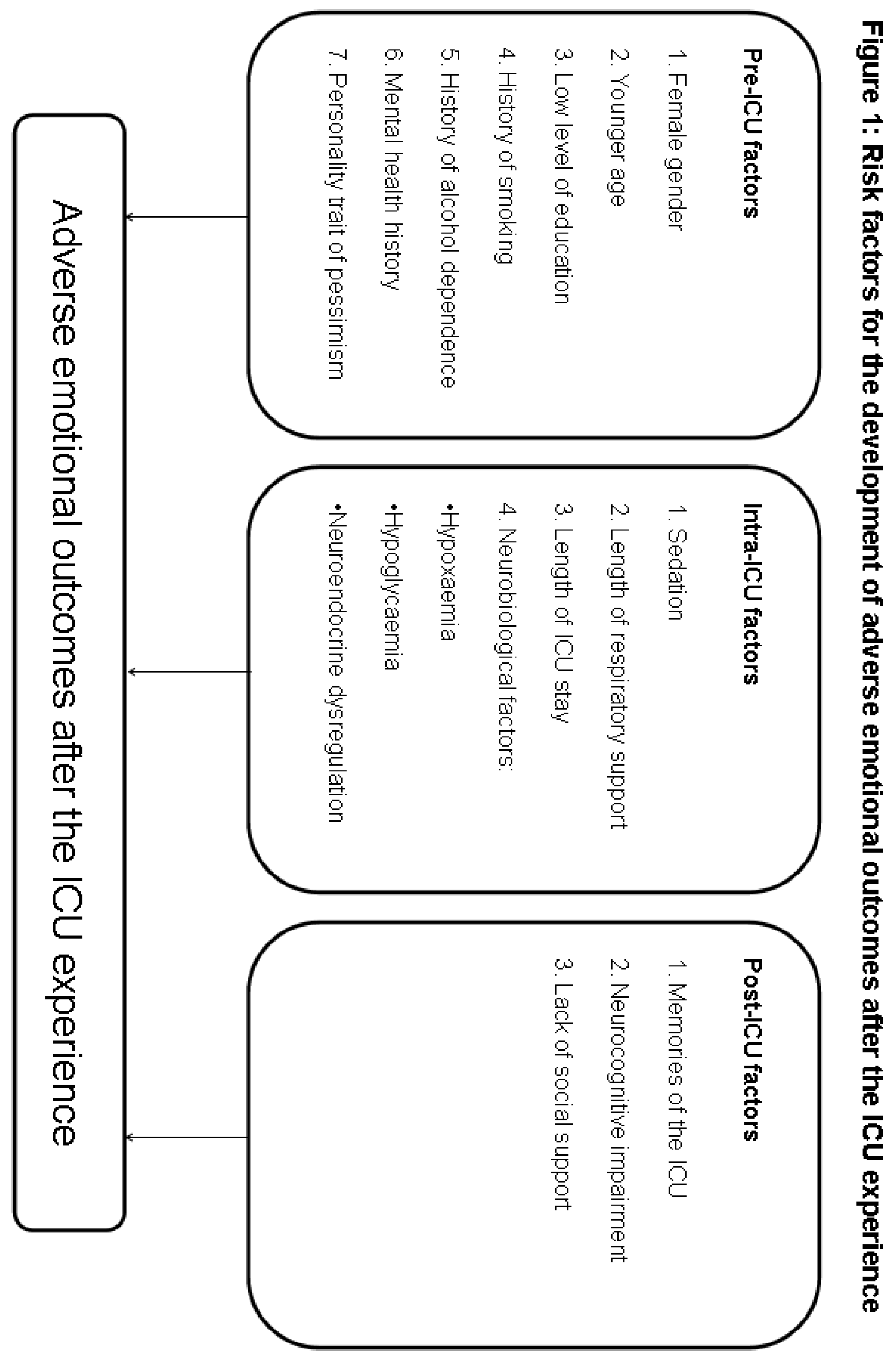

